\title{
Epsilon-near-zero media coupled with localized surface plasmon modes
}

\author{
Bilge Can Yildiz and Humeyra Caglayan (1)* \\ Faculty of Engineering and Natural Sciences, Photonics, Tampere University, 33720 Tampere, Finland
}

(Received 16 May 2020; revised 24 August 2020; accepted 11 September 2020; published 5 October 2020)

\begin{abstract}
Epsilon-near-zero (ENZ) media are an emerging platform, embracing the great potential for novel nanophotonic phenomena. One method to obtain an ENZ medium is to operate at the cutoff wavelength of the fundamental mode of a plasmonic waveguide. Control over this mode is limited to the waveguide's material and size properties. Here, we demonstrate that a plasmonic nanostructure (nanorod) can be strongly coupled to the plasmonic waveguide, providing two new hybrid resonance modes exhibiting characteristics of both guided ENZ modes and localized surface plasmon modes. Strong coupling gives rise to a Rabi splitting of $300 \mathrm{meV}$, which is demonstrated by finite-difference time domain simulations where we calculate the decay rate enhancement of a dipole emitter located in the coupled system. The hybrid modes are retrieved using the analytic coupled harmonic oscillator model. This suggested method via hybridization of modes can be used to generate and manipulate ENZ media where the unique ENZ property of wavelength extension enables effectively shrinking spatially long distances down to optically short distances.
\end{abstract}

DOI: 10.1103/PhysRevB.102.165303

\section{INTRODUCTION}

Recently, there has been a lot of interest in epsilonnear-zero (ENZ) materials, a new class of materials having vanishing permittivity at a certain frequency range [1-3]. Properties that are unique to ENZ media have enabled the realization of advanced optical applications such as directional light enhancement [4,5], coherent perfect absorption [6,7], radiation pattern tailoring [8], nonlinear fast optical switching [9], and index of refraction sensing [10].

The real part of the permittivity of many different materials vanishes in different spectral regions. For instance, noble metals such as gold and silver have ENZ wavelengths in the ultraviolet region, and yet at these wavelengths their imaginary epsilon is very high, which prevents making use of the ENZ properties of metals. Transparent conducting oxides (TCOs), such as indium tin oxide and aluminum zinc oxide, exhibit a dielectric to metallic transition around the telecom wavelength, where their real permittivity vanishes and the imaginary permittivity gets very close to zero. These materials are very promising for novel nanophotonic phenomena, such as enhancement of nonlinearities [11-13]. With the recent developments in artificially engineered materials, it has been possible to obtain and tailor effective ENZ media [13-15].

Apart from naturally occurring ENZ materials such as TCOs, there are several engineering approaches to achieve metamaterials and structures with ENZ properties. One way is to stack dielectric and metal subwavelength layers alternatingly on top of each other $[13,16]$, so that the overall

\footnotetext{
*humeyra.caglayan@tuni.fi
}

Published by the American Physical Society under the terms of the Creative Commons Attribution 4.0 International license. Further distribution of this work must maintain attribution to the author(s) and the published article's title, journal citation, and DOI. material dispersion displays effectively an epsilon-near-zero region at desired wavelengths, depending on material composition. There are also techniques to have mode dispersion with an ENZ region, rather than material dispersion. In the deep subwavelength-thickness limit, metal or TCO films were recently demonstrated to support so-called ENZ modes [17,18]. These are essentially a special case of long-range surface plasmon polariton modes in which the mode frequency $\omega$ is equal to the plasma frequency $\omega_{p}$ for a wide range of wave numbers, which corresponds to the electric permittivity, $\varepsilon=0$, very simply within the framework of the Drude model $\left[\varepsilon=1-\omega_{p}^{2} /\left(\omega^{2}+i \gamma \omega\right)\right]$.

Another technique based on structural dispersion instead of material dispersion is to exploit dispersion characteristics of composite metal-dielectric waveguides. Nanoscale plasmonic waveguides support a fundamental guided mode with a cutoff wavelength where the mode effective index vanishes, effectively generating an ENZ medium [19]. A rectangular waveguide that can support an ENZ mode can be fabricated using a bottom-up approach with a dielectric core. It is also possible to integrate this waveguide with a nanostructure embedded in the dielectric core. This ENZ medium supported by the waveguide mode provides a remarkable platform to implement practical applications of spontaneous emission enhancement [20,21]. Light propagation in the ENZ media has the property of a stretched wavelength as a consequence of the near-zero refractive index, leading to a relatively small phase variation over the physically large region of such media. Therefore, it is possible to effectively shrink spatially long distances down to optically short distances, which might be very useful for the interaction of quantum emitters at long distances, as previously proposed [22].

The operating wavelength of a plasmonic ENZ waveguide is determined by the geometrical and optical properties of its components, and hence, the tunability is limited to those properties. Here, we introduce a powerful method to manipulate the ENZ medium supported by a plasmonic nanoscale 

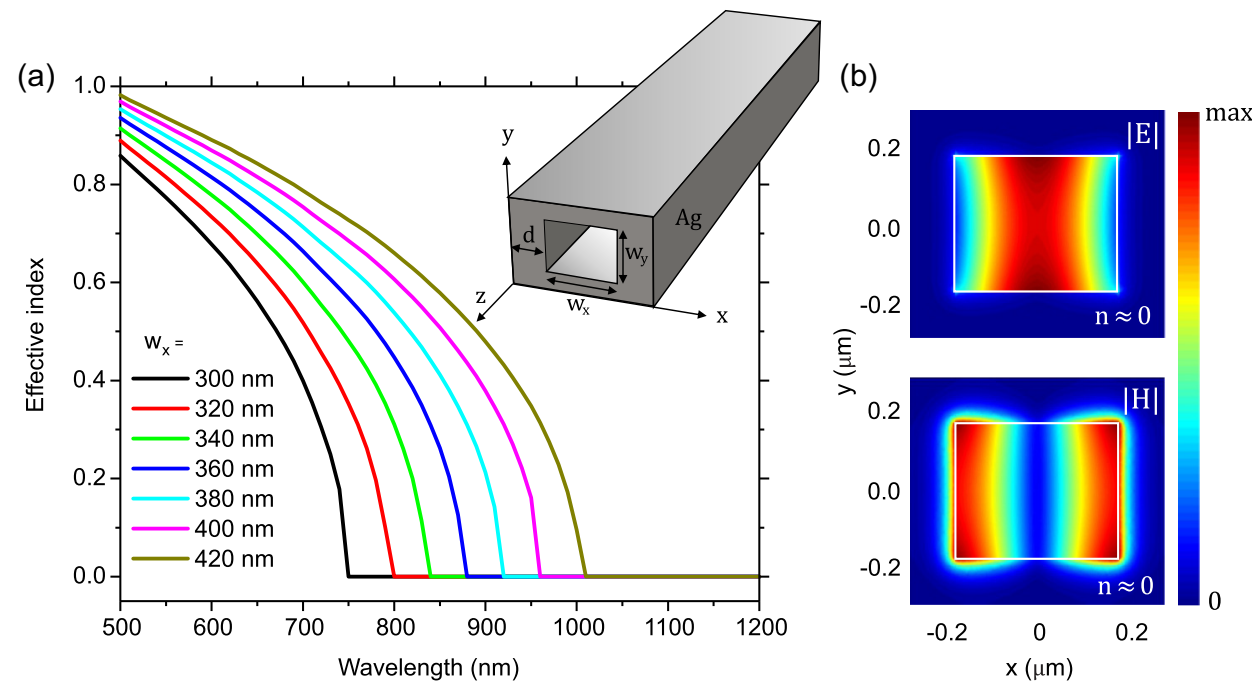

(c)

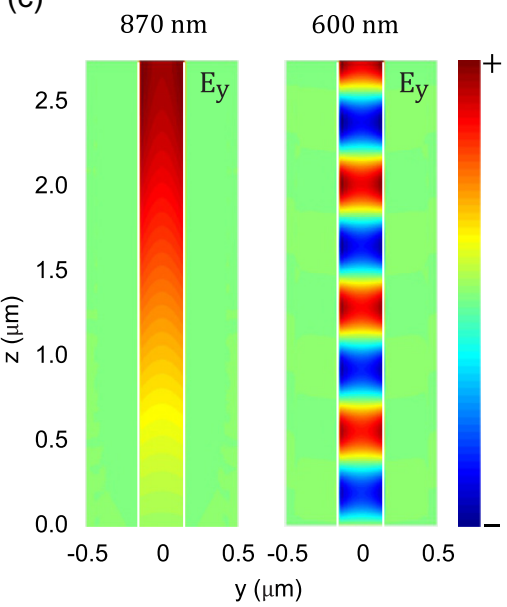

FIG. 1. (a) Dispersion curves of the fundamental mode for different core sizes $w_{x}$, with $w_{y}=300 \mathrm{~nm}$ and $d=200 \mathrm{~nm}$ for an infinite rectangular hollow silver waveguide, obtained in finite-element simulations. The inset shows the schematics. (b) Electric and magnetic field profiles at the $x y$ plane, at the cutoff wavelength $(870 \mathrm{~nm})$ of the waveguide with $w_{x}=360 \mathrm{~nm}$. (c) The $y$ component of the electric field along the waveguide axis at the $y z$ plane at the ENZ wavelength $(870 \mathrm{~nm})$ and at a shorter random wavelength $(600 \mathrm{~nm})$ with a nonzero index. White lines indicate the core borders.

waveguide. The strong-coupling phenomenon has been exploited in the sense that it enables making use of the individual features of individual modes in hybrid modes simultaneously, accompanied by an increased degree of freedom to tailor the resulting modes. For instance, planar ENZ modes supported by thin TCO films were previously demonstrated to strongly couple with localized/propagating surface plasmon modes, where the resulting hybrid modes brought advantages over the individual modes in the absence of coupling between them [23,24].

In this work, we theoretically investigate coupling properties of ENZ modes supported by plasmonic waveguides with localized surface plasmon modes. We demonstrate, as a proof of concept, that the fundamental $\left(\mathrm{TE}_{10}\right)$ mode of a rectangular plasmonic waveguide strongly couples with the localized surface plasmon resonance of a metal nanorod placed in the core of the waveguide at its cutoff wavelength, resulting in two hybrid modes. These modes exhibit mixed-field profiles of both ENZ and dipolar localized surface plasmons and provide enhanced density of optical states, demonstrated by the decay rate enhancement of a dipole emitter located near the nanorod.

\section{FUNDAMENTAL MODE OF THE PLASMONIC WAVEGUIDE AT THE CUTOFF}

We design an ENZ medium by taking advantage of a particular dispersion relation between the wavelength and wave number in a hollow rectangular metallic nanoscale waveguide. The fundamental $\left(\mathrm{TE}_{10}\right)$ mode of such waveguides possesses a dispersion curve such that it starts near the light line and ends up cutting off the wavelength axis at $k=0$. In contrast, the dispersion curve of a normal dielectric waveguide crosses the light line (of the low-index cladding medium), corresponding to a cutoff at a nonzero wave number, below which light leaks out, where the mode is no longer supported. A vanishingly small wave vector at the cutoff wavelength indicates a waveguide mode with near-zero index $\left(n_{\text {eff }}=k / k_{0}\right)$, as well as a stretched wavelength $\left(\lambda=\lambda_{0} / n_{\text {eff }}\right)$ approaching infinity, where the wave exhibits little or no spatial variation. The inset in Fig. 1(a) shows the schematics of the designed plasmonic waveguide. It is a rectangular hollow waveguide with edges $w_{x}$ and $w_{y}$ and a silver cladding of thickness $d=200 \mathrm{~nm}$ embedded in air. We keep one edge of the core fixed, $w_{y}=300$ $\mathrm{nm}$, and control the cutoff wavelength by changing the other edge, $w_{x}=300-420 \mathrm{~nm}$. We calculate the dispersion of the waveguide by the finite-element method, using Finite Element EigenMode (FEEM), a commercially available solver provided by Lumerical Inc. The structure under consideration is infinitely long and uniform along the waveguide $(z)$ axis. Hence, the characteristic solutions or the waveguide modes have a spatial dependency of the form $e^{i \beta z}$, where $\beta$ is the propagation constant (the wave number of the mode to be solved). In the absence of sources, Maxwell's equations are given by

$$
\begin{aligned}
i \omega \mu \boldsymbol{H} & =\boldsymbol{\nabla} \times \boldsymbol{E}, \\
i \omega \varepsilon \boldsymbol{E} & =-\nabla \times \boldsymbol{H},
\end{aligned}
$$

where $\mu$ and $\varepsilon$ are the permeability and permittivity, respectively, and $\omega$ is the angular frequency. The electromagnetic fields have spatial dependence in the following form:

$$
\begin{aligned}
& \boldsymbol{E}(x, y, z)=\boldsymbol{E}(x, y) e^{i \beta z}, \\
& \boldsymbol{H}(x, y, z)=\boldsymbol{H}(x, y) e^{i \beta z} .
\end{aligned}
$$

Substituting these into Maxwell's equations, we eliminate the $z$ dependence, and the problem reduces to a two-dimensional coupled eigenvalue problem, which we numerically solve to obtain the dispersion relation, which is a set of effective index $n_{\text {eff }}$ vs wavelength $\lambda$ values, where $n_{\mathrm{eff}}=(c / \omega) \beta$, with $c$ being the speed of light, as well as the mode field profiles. The dispersion curves of the fundamental $\left(\mathrm{TE}_{10}\right)$ mode of the waveguide for different core sizes $w_{x}$ is shown in Fig. 1(a). The waveguide exhibits cutoff 
wavelengths in the range from 750 to $1050 \mathrm{~nm}$, with corresponding effective index $n_{\text {eff }}=0$. The cutoff wavelength shifts to longer wavelengths as the core size increases, as expected. The mode profiles at the cross-section $(x y)$ plane of the waveguide at the cutoff wavelength $(870 \mathrm{~nm})$ for a core size of $360 \times 300 \mathrm{~nm}^{2}$ are shown in Fig. 1(b). The electric field profile shows that the mode is strongly enhanced around the upper and lower walls of the waveguide corresponding to a $y$-polarized mode. We note that the nearest higher-order mode supported by this waveguide is in the ultraviolet regime [21]. To obtain the mode profiles along the waveguide axis, we employ the finite-difference time domain (FDTD) method to solve Maxwell's equations in three dimensions, using Lumerical FDTD Solutions. The length of the waveguide is set approximately ten times larger than the core size (a few microns) to ensure uniformity along the waveguide axis. We use a broadband Mode source to inject the fundamental guided mode, which is the $\mathrm{TE}_{10}$ mode for a rectangular waveguide. It allows us to obtain the field profiles at all wavelengths within the source range. We present the $y$-component electric field along the waveguide axis at the $y z$ plane at the cutoff wavelength in Fig. 1(c), and we also plot this profile at a random wavelength shorter than the cutoff, $600 \mathrm{~nm}$, for comparison. It is instructive to observe that the electric field does not experience much phase variation along the waveguide at the cutoff wavelength, where the effective index is close to zero. However, when the wavelength is shorter than the cutoff, it exhibits a regular guided mode behavior. It is also worth noting that the waveguide mode at the cutoff offers a propagation length of about two times the free-space wavelength [25].

\section{DECAY RATE ENHANCEMENT IN THE ENZ REGIME}

We observe that at the cutoff wavelength, the mode profile along the waveguide axis exhibits almost no spatial variations (where the wavelength $\lambda=\lambda_{0} / n_{\text {eff }}$ approaches infinity), which is a unique property of electromagnetic oscillations within the ENZ media [Fig. 1(c)]. The property of stretching the wavelength makes the plasmonic waveguides attractive for energy squeezing and tunneling $[2,26]$ and observation of the collective optical response of quantum emitters such as strong dipole-dipole interactions, entanglement, and superradiance [20,21,27-29]. Hence, we study the decay properties of a dipole emitter located in the core of the plasmonic waveguide and observe how the dipole emitter couples to the waveguide mode for different core sizes as a function of wavelength. The spontaneous decay rate of a dipole emitter located at a point $\boldsymbol{r}_{\mathbf{0}}$ is given by

$$
\gamma=\frac{2 \omega}{3 \hbar \varepsilon_{0}}|\boldsymbol{p}|^{2} \rho\left(\boldsymbol{r}_{\mathbf{0}}, \omega\right)
$$

where $\boldsymbol{p}$ is the transition dipole moment and $\rho\left(\boldsymbol{r}_{\mathbf{0}}, \omega\right)$ is the partial local density of states, which is defined by

$$
\rho\left(\boldsymbol{r}_{\mathbf{0}}, \omega\right)=\frac{6 \omega_{0}}{\pi c^{2}}\left[\boldsymbol{n} \cdot \operatorname{Im}\left\{\overline{\boldsymbol{G}}\left(\boldsymbol{r}_{\mathbf{0}}, \boldsymbol{r}_{\mathbf{0}}, \omega\right)\right\} \cdot \boldsymbol{n}\right] .
$$

Here, $\boldsymbol{n}$ is the unit vector along the dipole orientation, and $\overline{\boldsymbol{G}}\left(\boldsymbol{r}, \boldsymbol{r}_{\mathbf{0}}, \omega\right)$ is the Green's dyadic, which is, in general, a $3 \times 3$ matrix, electromagnetically quantifying the point $\boldsymbol{r}$ due to the presence of a point source located at $\boldsymbol{r}_{\mathbf{0}}$ at the frequency $\omega$. We note that since the fundamental mode profile of the waveguide is polarized along the $y$ direction, it does not support modes to which $x$ - and $z$-polarized dipole emitters could couple in the wavelength region of interest, and hence, we consider only the interactions between $y$-oriented dipole emitters and corresponding electric field components. Specific to our discussion here, the compact relation between the Green's dyadic and the electric field at the position of the dipole emitter $E_{y}$ is given by

$$
G_{y}=\frac{\varepsilon_{0} \varepsilon c^{2}}{p \omega^{2}} E_{y} .
$$

Here, $p$ is the dipole moment of the $y$-oriented dipole emitter, and $E_{y}$ is the electric field generated by the local dipole source. We use Lumerical FDTD Solutions to compute $E_{y}$ fields within the waveguide core excited by the dipole emitter located in the core of the waveguide. We employ a large three-dimensional FDTD simulation region ( $5 \mu \mathrm{m}$ in the $z$ direction) in which the waveguide extends throughout the $z$ boundaries along its axis. The boundary conditions are set to perfectly matched layers in all directions. Silver is modeled by the tabulated experimental dielectric function provided by Palik [30]. The background region as well as the core domain is air $(n=1)$. The dipole emitter source is placed at the center of the waveguide such that its polarization is along the $y$ axis. The emitter used in the calculations is an oscillating dipole which is commonly used to simulate point source radiators, such as radiation from a fluorescent molecule. A power box monitor enclosing the dipole emitter source (as well as the metal nanorod for the cases where the nanorod is present) was used to calculate the power radiated by the dipole emitter to obtain the decay rate enhancement. A finer mesh than the default (mesh accuracy 3 ) is applied to the whole FDTD domain, whereas the mesh near the dipole and the nanorod is overwritten such that the smallest element is $4 \mathrm{~nm}$ to better resolve the region of interest. We then substitute $E_{y}$ fields obtained in FDTD simulations in Eq. (5) to obtain the green dyadic and then substitute the green dyadic in Eq. (4) to obtain the partial density of states and then finally substitute the partial density of states in Eq. (3) to obtain the decay rate enhancement as a function of wavelength for waveguides with different core sizes (and hence different cutoff wavelengths). Figure 2 shows the decay rate enhancement of the dipole emitter (decay rate of the dipole emitter in the presence of the waveguide divided by the decay rate in free space), calculated using Eq. (3). We observe the decay rate enhancement peaks at the cutoff wavelength of each waveguide and then abruptly drops to zero, as expected. In fact, calculating the decay rate enhancement of a dipole emitter located in the core of a plasmonic waveguide is a way to obtain the ENZ wavelength of that waveguide. We adopt these calculations to understand the optical response of the waveguide coupled to a plasmonic nanorod in the next section.

\section{COUPLED WAVEGUIDE-PLASMON MODES}

In this section, we present the optical response of the plasmonic ENZ waveguide when a metal nanorod is placed in the core in terms of how the decay rate enhancement of the dipole emitter is modified. A gold rectangular nanorod with a 


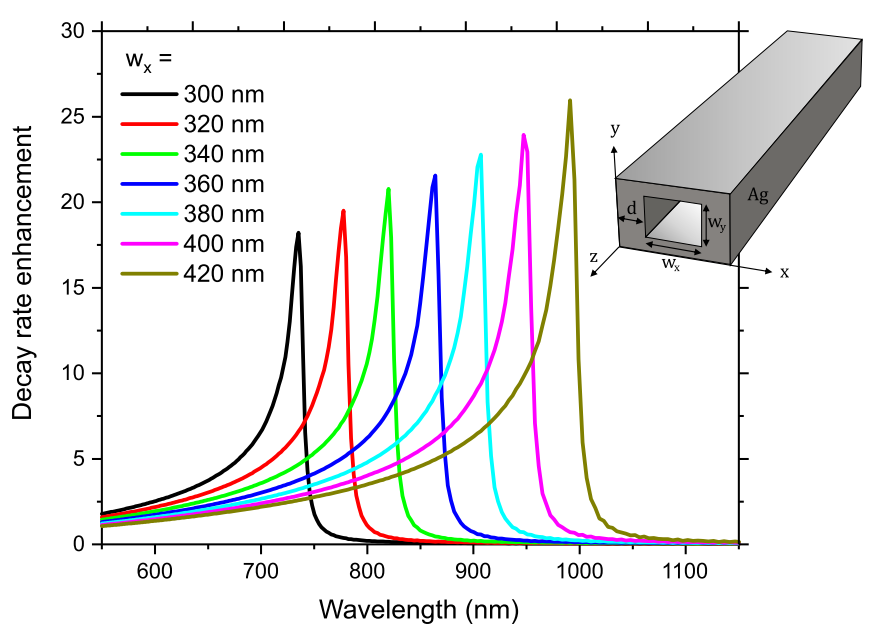

FIG. 2. Decay rate enhancement of a $y$-oriented dipole emitter located at the core of the waveguide with different core sizes $w_{x}$ for $w_{y}=300 \mathrm{~nm}$ and $d=200 \mathrm{~nm}$ kept fixed. The inset shows the schematics of the waveguide.

long-axis length $L=140 \mathrm{~nm}$ and short edges of $20 \times 20 \mathrm{~nm}^{2}$ is located at the center of the waveguide core, such that its long axis is along the $y$ axis. The local dipole source is located in the waveguide core too, $15 \mathrm{~nm}$ away from the nanorod, oriented along the $y$ axis, as shown in the inset schematics of Fig. 3. Figure 3(a) shows the decay rate enhancement of the dipole emitter as a function of wavelength for the different waveguide core sizes, $w_{x}=300-420 \mathrm{~nm}$. The vertical axis is the offset decay rate enhancement, which means each curve is shifted vertically to provide better visualization of how the hybrid resonances change for different waveguide dimensions. We observe two resonances that exhibit anticrossing (dashed lines), suggesting hybridization between two optical modes: the localized surface plasmon mode and the fundamental guided mode. The anticrossing behavior is more obvious in Fig. 3(b), where we plot the decay rate enhancement of the dipole emitter for a fixed waveguide size $\left(w_{x}=360 \mathrm{~nm}\right.$, $w_{y}=300 \mathrm{~nm}$ ) but a changing nanorod length in the range $L=80-200 \mathrm{~nm}$. As the vertical scale is squeezed a lot in these displays, the curves appear to be flat. We note that the peak values of the resonances reach up to $\sim 120$ and the lowest peak value is $\sim 20$; that is, the obtained decay rate enhancement in the presence of the nanorod is either comparable to or five to six times larger than the one in the absence of the nanorod. At the anticrossing point, corresponding to the configuration shown by the blue curves in Fig. $3\left(w_{x}=360 \mathrm{~nm}\right.$ and $L=140 \mathrm{~nm}$ ), the energy split is $300 \mathrm{meV}$ (resonance wavelengths of 780 and $940 \mathrm{~nm}$ ), and the full width at half maximum (FWHM) of the broader resonance is $245 \mathrm{meV}$. Since the energy split is higher than the FWHM of the lowestquality resonance, we conclude that the localized plasmon mode of the nanorod and guided mode supported by the ENZ waveguide are hybridized in the strong-coupling regime [31].

To have a clearer picture of the hybridization, we compare the uncoupled modes and the hybrid modes over the anticrossing scheme. The (uncoupled) localized surface plasmon resonances are obtained similarly by calculating the decay rate enhancement of a local dipole source near the nanorod in free space based on the fact that surface plasmons give rise to the enhancement of the local density of states at the plasmon resonance. We note that we obtain the same optical response spectra by calculating the scattering cross section of the gold nanorod excited by a linearly polarized plane wave. Figure 4(a) shows a color map of the decay rate enhancement of a dipole emitter located in the configuration shown in the inset of Fig. 3(b) as a function of wavelength and the nanorod size ranging between $L=80$ and $200 \mathrm{~nm}$ for fixed $w_{x}=360 \mathrm{~nm}$ and $w_{y}=300 \mathrm{~nm}$. White circles show the uncoupled localized surface plasmon resonances obtained in the calculations of the decay rate enhancement of the dipole emitter near the nanorod in free space. The uncoupled fundamental guided mode of the ENZ waveguide is shown by white squares at $870 \mathrm{~nm}$. The decay rate enhancement spectra of the

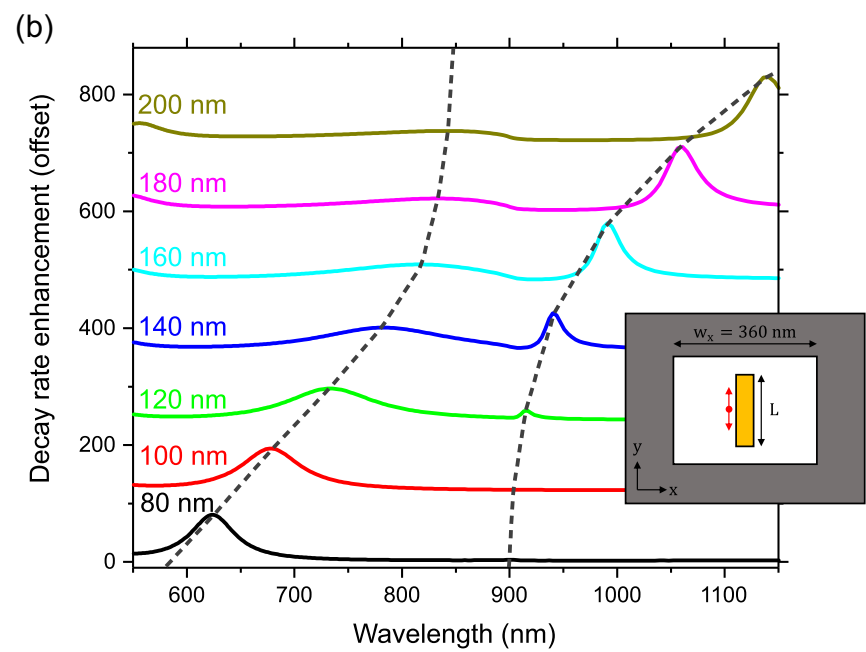

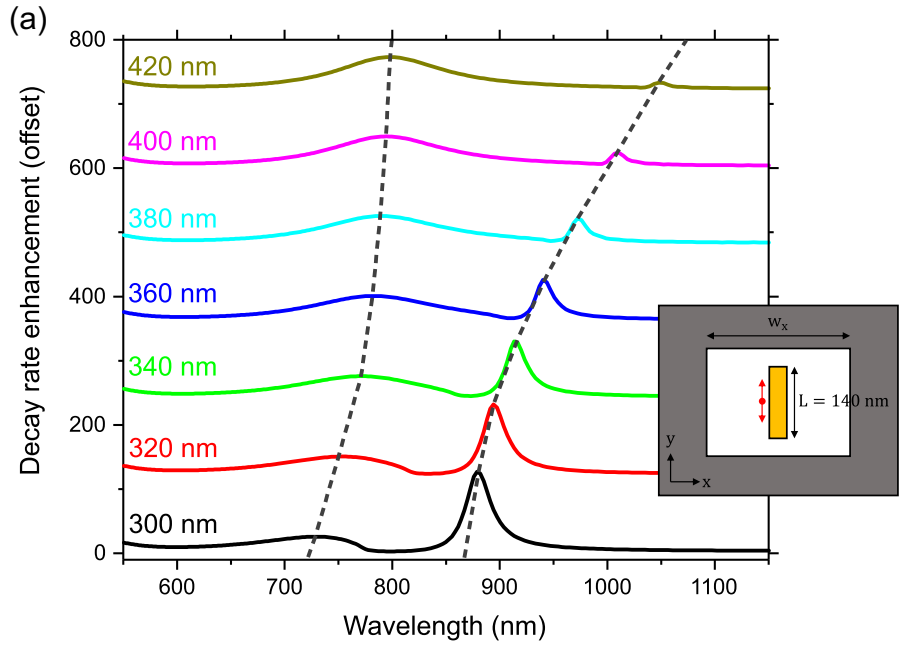

FIG. 3. Decay rate enhancement spectra for the coupled waveguide-nanorod system. Each spectrum is shifted vertically to provide better visualization. (a) Each color stands for a different waveguide size. $w_{x}$ ranges between 300 and $420 \mathrm{~nm}$, while $w_{y}=300 \mathrm{~nm}$ and the length of the gold nanorod $L=140 \mathrm{~nm}$ are kept fixed. (b) Each color stands for a different nanorod length. $L$ ranges between 80 and $200 \mathrm{~nm}$ for a fixed ENZ waveguide with $w_{x}=360 \mathrm{~nm}$ and $w_{y}=300 \mathrm{~nm}$. 

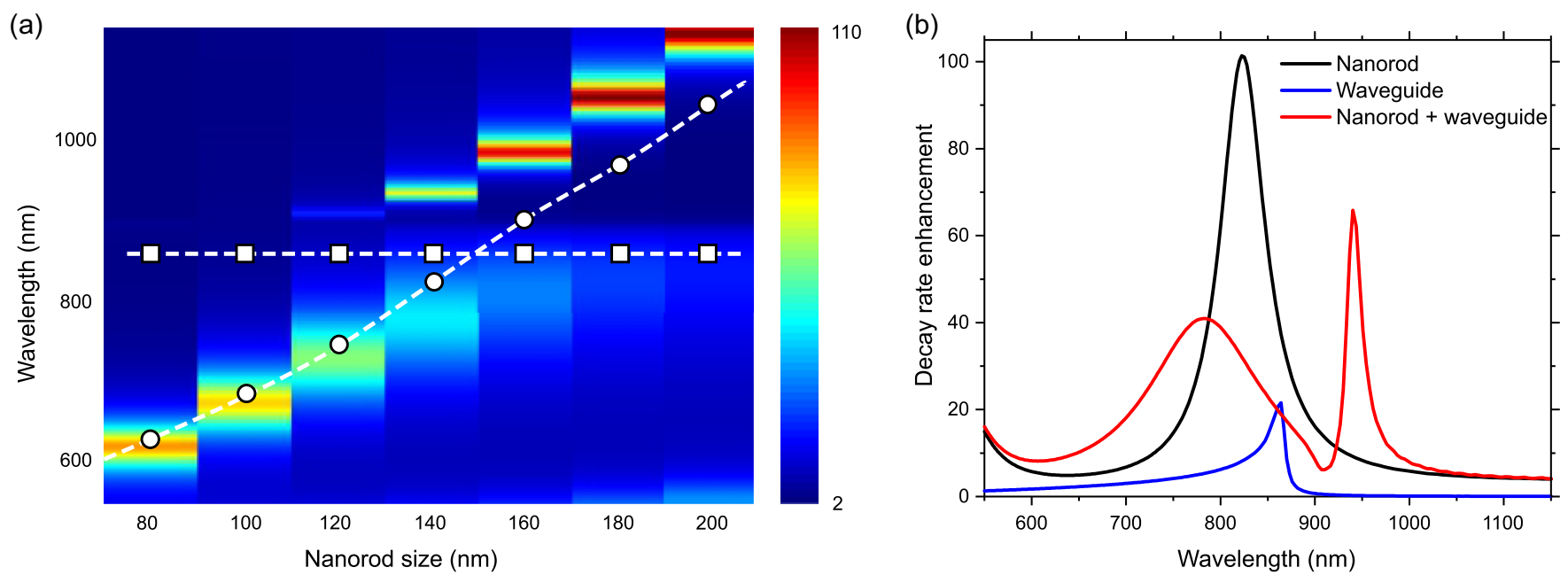

FIG. 4. (a) Decay rate enhancement spectra for the coupled waveguide-nanorod system as a function of wavelength and nanorod size for $w_{x}=360 \mathrm{~nm}$ and $w_{y}=300 \mathrm{~nm}$. White circles and white squares show the uncoupled localized surface plasmon resonances and uncoupled fundamental waveguide mode, respectively. (b) Spectra for the uncoupled and coupled modes for $L=140 \mathrm{~nm}$, $w_{x}=360 \mathrm{~nm}$, and $w_{y}=300 \mathrm{~nm}$.

coupled system follow the uncoupled mode wavelengths at the points far from the anticrossing, but the resonance is split well at the anticrossing point for $L \sim 140 \mathrm{~nm}$. In Fig. 4(b), we plot the decay rate enhancement spectra of the coupled and uncoupled systems for the anticrossing configuration. For $L=140 \mathrm{~nm}$ and $w_{x}=360 \mathrm{~nm}$, the uncoupled modes overlap around the 850-880-nm wavelength region. When these two systems both supporting uncoupled modes are brought together upon excitation by a local dipole source, we observe two distinct, pronounced resonance modes at 780 and $940 \mathrm{~nm}$, rather than the superposition of the two modes (where the two uncoupled modes are added up) [32,33]. The superposition scenario applies when the uncoupled modes are spectrally far from each other. For example, when the nanorod size $L=80 \mathrm{~nm}$, the uncoupled modes and the modes arising from the coupled overall system spectrally overlap (at approximately 610 and $870 \mathrm{~nm}$ ). For smaller nanorods, the waveguide mode is barely visible; the dipole source dominantly couples with the localized surface plasmon mode on the nanorod. For longer nanorods $(L=180-200 \mathrm{~nm})$, the hybrid resonances at the shorter wavelengths spectrally overlap well with the uncoupled waveguide mode, whereas the hybrid resonances at longer wavelengths are redshifted from the uncoupled localized surface plasmon modes. The reason for the latter might be that when the nanorod gets longer, it starts coupling with the waveguide's metal cladding.

Next, we use the coupled harmonic oscillator model to retrieve the hybrid resonances of the coupled ENZ-plasmon system. To model this system, we consider two harmonic oscillators with frequency-dependent amplitudes $x_{1}(\omega)$ and $x_{2}(\omega)$, resonance frequencies $\omega_{1}$ and $\omega_{2}$, and damping rates $\gamma_{1}$ and $\gamma_{2}$. The two oscillators are coupled under the two harmonic driving forces $f_{1}(\omega)$ and $f_{2}(\omega)$, with a coupling constant $g$. The equations of motion of such a system are written as

$$
\left[\begin{array}{cc}
\omega_{1}-\omega-i \gamma_{1} & g \\
g & \omega_{2}-\omega-i \gamma_{2}
\end{array}\right]\left[\begin{array}{l}
x_{1}(\omega) \\
x_{2}(\omega)
\end{array}\right]=\left[\begin{array}{l}
f_{1}(\omega) \\
f_{2}(\omega)
\end{array}\right]
$$

This system of equations can be solved to find the eigenvalues by setting the determinant of the $2 \times 2$ matrix to zero. The resulting expression for the eigenvalues $\omega^{(+)}$and $\omega^{(-)}$(upper and lower hybrid resonances) is the following:

$$
\begin{aligned}
\omega^{( \pm)}= & \frac{1}{2}\left[\omega_{1}+\omega_{2}-i\left(\gamma_{1}+\gamma_{2}\right)\right] \\
& \pm \frac{1}{2} \sqrt{\left[\omega_{1}-\omega_{2}-i\left(\gamma_{1}-\gamma_{2}\right)\right]^{2}+4 g^{2}},
\end{aligned}
$$

where the real parts determine the spectral locations and imaginary parts dictate the linewidths.

We substitute the uncoupled mode frequencies [white circles and squares in Fig. 4(a)] and the value of the Rabi splitting $\left(2 g=\hbar \Omega_{R}=300 \mathrm{meV}\right)$ obtained in the FDTD simulations. Figure 5(a) show the full hybridization picture obtained from the FDTD simulations and reproduced by the analytical coupled harmonic oscillator model. In Fig. 5(a), resonances obtained in the FDTD simulations are shown by symbols, whereas the ones obtained by the coupled harmonic oscillator model are shown by the red solid lines. We present the resonance wavelengths of coupled and uncoupled modes with respect to the nanorod size. Black squares and blue circles show the uncoupled localized surface plasmon mode resonances and the uncoupled waveguide mode resonances, respectively, whereas the red triangles show the upper and lower hybrid mode resonances. Here, the terms upper and lower conventionally refer to the energy (or frequency) but not the wavelength, so the upper hybrid band in Fig. 5(a) appears at the lower wavelengths and vice versa.

Strong hybridization between the localized surface plasmons and the waveguide mode at the cutoff wavelength of the plasmonic waveguide provides two new optical modes with the characteristics of both of the uncoupled modes. We present electric field profiles calculated at the upper and lower hybrid mode resonance wavelengths, 780 and $940 \mathrm{~nm}$, obtained in the coupled system in Fig. 5(b). We use a y-polarized plane wave initiated at one end of the waveguide, propagating along the waveguide axis ( $z$ direction) towards the gold nanorod located at the center of the waveguide. We obtain the $E_{y}$ profile at 

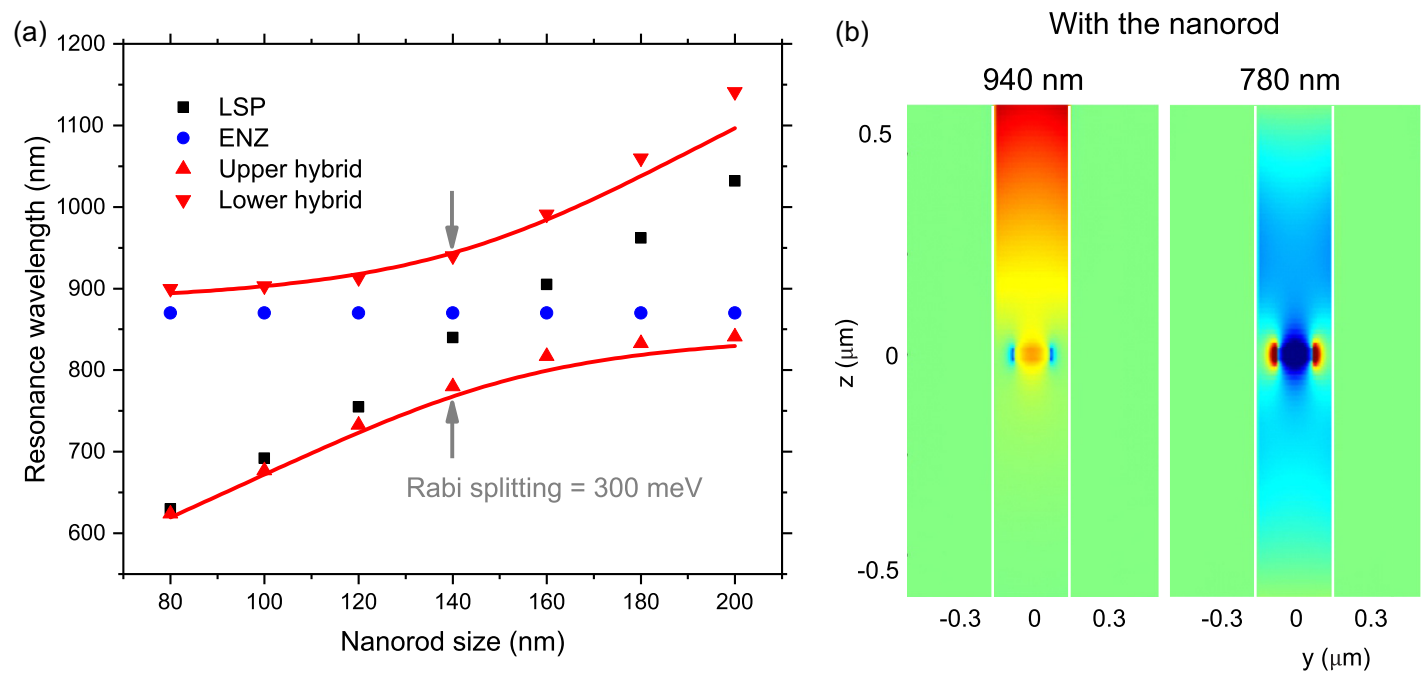

Without the nanorod

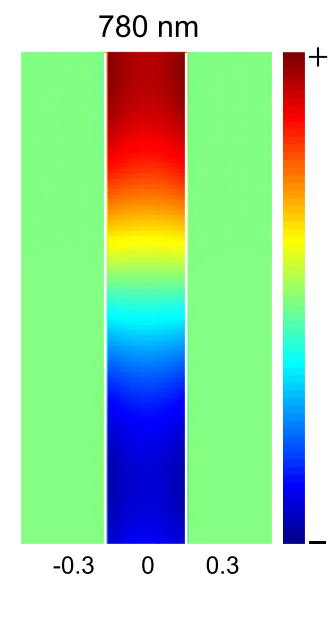

FIG. 5. (a) Complete hybridization scheme, showing the resonance wavelengths of the coupled hybrid modes (red) and uncoupled ENZ waveguide and localized surface plasmon (LSP) modes (blue and black, respectively), calculated in FDTD simulations (symbols) and the coupled harmonic oscillator model (solid lines), with respect to the nanorod length $L$ for $w_{x}=360 \mathrm{~nm}$ and $w_{y}=300 \mathrm{~nm}$. (b) The $y$ component of the electric field at the lower and upper hybrid mode resonances, 940 and $780 \mathrm{~nm}$ (left and middle, respectively), for the coupling configuration and the waveguide alone at $780 \mathrm{~nm}$ (right) for comparison. White lines indicate the core borders.

the $y z$ plane. At $940 \mathrm{~nm}$, the field profile exhibits a dipolar distribution as a result of charge alignment along the nanorod axis upon excitation of the localized surface plasmon mode, and a constant phase background field is observed as a result of the ENZ characteristic of the waveguide mode. At $780 \mathrm{~nm}$, similar to the other hybrid mode, there are both plasmon and ENZ characteristics in the field profile. It is worth noting that at $780 \mathrm{~nm}$, we observe phase modulation (i.e., the electric field oscillates along the $z$ axis) when observing from a longer $z$ scale; however, the presence of the nanorod at the center of the waveguide modifies the background guided field such that it has a constant phase within the spatial coupling region. As a comparison, we also present the field profile calculated in the absence of the coupling, i.e., for the case without the nanorod, at the same wavelength, $780 \mathrm{~nm}$.

\section{DISCUSSION AND CONCLUSIONS}

In summary, we studied coupling between the fundamental mode of a rectangular plasmonic waveguide providing an ENZ medium at its cutoff wavelength and the localized plasmon resonance of a gold nanorod located at the center of the waveguide. We calculated the decay rate enhancement of a dipole emitter near this system to determine the coupled and uncoupled modes in the FDTD simulations. The uncoupled waveguide mode is controlled by the waveguide core size, whereas the uncoupled localized surface mode is controlled by the nanorod length. For a certain nanorod length and waveguide core size, the uncoupled optical spectra of the resonance modes overlap. We demonstrated that these modes are strongly coupled by comparing the Rabi splitting and the linewidths of the waveguide and plasmon modes. We also showed that the coupling between the waveguide ENZ mode and the plasmon mode agrees well with the coupled harmonic oscillator model, where the hybrid mode resonances are reproduced. The obtained hybrid modes exhibit characteristics of both uncoupled modes. That is, at the hybrid mode resonances, the field distributions display a mixture of dipolar plasmons and constant-phase guided profile. This coupling phenomenon provides a powerful method to control the resonance modes supported by waveguides, where, otherwise, the only way to tune the cutoff wavelength is to change the dimensions or materials. Employing a plasmonic nanostructure simply brings further control over the optical response, enabling two instead of one ENZ region as well as further degrees of freedom, i.e., the dimension, shape, and material of the plasmonic nanostructure.

\section{ACKNOWLEDGMENTS}

We acknowledge the financial support of the H2020 European Research Council (Starting Grant project aQUARiUM; Agreement No. 802986), Academy of Finland Flagship Programme (PREIN; Grant No. 320165).
[1] N. Engheta, Pursuing near-zero response, Science 340, 286 (2013).

[2] M. G. Silveirinhaand N. Engheta, Theory of supercoupling, squeezing wave energy, and field confinement in narrow channels and tight bends using $\epsilon$ near-zero metamaterials, Phys. Rev. B 76, 245109 (2007).
[3] N. Kinsey, C. DeVault, A. Boltasseva, and V. M. Shalaev, Near-zero-index materials for photonics, Nat. Rev. Mater. 4, 742 (2019).

[4] H. Hajian, E. Ozbay, and H. Caglayan, Beaming and enhanced transmission through a subwavelength aperture via epsilonnear-zero media, Sci. Rep. 7, 4741 (2017). 
[5] H. Hajian, E. Ozbay, and H. Caglayan, Enhanced transmission and beaming via a zero-index photonic crystal, Appl. Phys. Lett. 109, 031105 (2016).

[6] Y. Li and C. Argyropoulos, Tunable nonlinear coherent perfect absorption with epsilon-near-zero plasmonic waveguides, Opt. Lett. 43, 1806 (2018).

[7] W. Ji, D. Wang, S. Li, Y. Shang, W. Xiong, L. Zhang, and J. Luo, Photonic-doped epsilon-near-zero media for coherent perfect absorption, Appl. Phys. A 125, 129 (2019).

[8] A. Alù, M. G. Silveirinha, A. Salandrino, and N. Engheta, Epsilon-near-zero metamaterials and electromagnetic sources: Tailoring the radiation phase pattern, Phys. Rev. B 75, 155410 (2007).

[9] G. A. Wurtz, R. Pollard, W. Hendren, G. P. Wiederrecht, D. J. Gosztola, V. A. Podolskiy, and A. V. Zayats, Designed ultrafast optical nonlinearity in a plasmonic nanorod metamaterial enhanced by nonlocality, Nat. Nanotechnol. 6, 107 (2011).

[10] A. R. Rashed, B. Gudulluoglu, H. W. Yun, I. H. B. M. Habib, S. H. Hong, E. Ozbay, and H. Caglayan, Highly-sensitive refractive index sensing by near-infrared metatronic nanocircuits, Sci. Rep. 8, 11457 (2018).

[11] M. Z. Alam, I. De Leon, and R. W. Boyd, Large optical nonlinearity of indium tin oxide in its epsilon-near-zero region, Science 352, 795 (2016).

[12] N. Kinsey, C. DeVault, J. Kim, M. Ferrera, V. M. Shalaev, and A. Boltasseva, Epsilon-near-zero Al-doped $\mathrm{ZnO}$ for ultrafast switching at telecom wavelengths, Optica 2, 616 (2015)

[13] A. R. Rashed, B. C. Yildiz, S. R. Ayyagari, and H. Caglayan, Hot electron dynamics in ultrafast multilayer epsilon-near-zero metamaterials, Phys. Rev. B 101, 165301 (2020).

[14] J. Gao, L. Sun, H. Deng, C. J. Mathai, S. Gangopadhyay, and X. Yang, Experimental realization of epsilon-near-zero metamaterial slabs with metal-dielectric multilayers, Appl. Phys. Lett. 103, 051111 (2013).

[15] R. Maas, J. Parsons, N. Engheta, and A. Polman, Experimental realization of an epsilon-near-zero metamaterial at visible wavelengths, Nat. Photonics 7, 907 (2013).

[16] T. Tumkur, Y. Barnakov, S. T. Kee, M. A. Noginov, and V. Liberman, Permittivity evaluation of multilayered hyperbolic metamaterials: Ellipsometry vs. reflectometry, J. Appl. Phys. 117, 103104 (2015).

[17] S. Campione, I. Brener, and F. Marquier, Theory of epsilonnear-zero modes in ultrathin films, Phys. Rev. B 91, 121408(R) (2015).

[18] S. Vassant, J.-P. Hugonin, F. Marquier, and J.-J. Greffet, Berreman mode and epsilon near zero mode, Opt. Express 20, 23971 (2012).
[19] E. J. R. Vesseur, T. Coenen, H. Caglayan, N. Engheta, and A. Polman, Experimental Verification of $n=0$ Structures for Visible Light, Phys. Rev. Lett. 110, 013902 (2013).

[20] R. Sokhoyan and H. A. Atwater, Cooperative behavior of quantum dipole emitters coupled to a zero-index nanoscale waveguide, arXiv:1510.07071.

[21] R. Sokhoyanand H. A. Atwater, Quantum optical properties of a dipole emitter coupled to an e-near-zero nanoscale waveguide, Opt. Express 21, 32279 (2013).

[22] A. M. Mahmoudand N. Engheta, Wave-matter interactions in epsilon-and-mu-near-zero structures, Nat. Commun. 5, 5638 (2014).

[23] E. L. Runnerstrom, K. P. Kelley, T. G. Folland, J. R. Nolen, N. Engheta, J. D. Caldwell, and J.-P. Maria, Polaritonic hybridepsilon-near-zero modes: Beating the plasmonic confinement vs propagation-length trade-off with doped cadmium oxide bilayers, Nano Lett. 19, 948 (2019).

[24] S. Campione, J. R. Wendt, G. A. Keeler, and T. S. Luk, Near-infrared strong coupling between metamaterials and epsilon-near-zero modes in degenerately doped semiconductor nanolayers, ACS Photonics 3, 293 (2016).

[25] R. Liu, C. M. Roberts, Y. Zhong, V. A. Podolskiy, and D. Wasserman, Epsilon-near-zero photonics wires, ACS Photonics 3, 1045 (2016).

[26] B. Edwards, A. Alù, M. E. Young, M. Silveirinha, and N. Engheta, Experimental Verification of Epsilon-Near-Zero Metamaterial Coupling and Energy Squeezing Using a Microwave Waveguide, Phys. Rev. Lett. 100, 033903 (2008).

[27] R. Fleury and A. Alù, Enhanced superradiance in epsilon near zero plasmonic channels, Phys. Rev. B 87, 201101(R) (2013).

[28] Y. Li, A. Nemilentsau, and C. Argyropoulos, Resonance energy transfer and quantum entanglement mediated by epsilon-nearzero and other plasmonic waveguide systems, Nanoscale 11, 14635 (2019).

[29] E. Özgün, E. Ozbay, and H. Caglayan, Tunable zero-index photonic crystal waveguide for two-qubit entanglement detection, ACS Photonics 3, 2129 (2016).

[30] E. D. Palik, Handbook of Optical Constants of Solids (Academic, San Diego, 1998).

[31] D. S. Dovzhenko, S. V. Ryabchuk, Y. P. Rakovich, and I. R. Nabiev, Light matter interaction in the strong coupling regime: Configurations, conditions, and applications, Nanoscale $\mathbf{1 0}$, 3589 (2018).

[32] A. P. Manuel, A. Kirkey, N. Mahdi, and K. Shankar, Plexcitonics - Fundamental principles and optoelectronic applications, J. Mater. Chem. C 7, 1821 (2019).

[33] N. Fain, T. Ellenbogen, and T. Schwartz, Near-field analysis of strong coupling between localized surface plasmons and excitons, Phys. Rev. B 100, 235448 (2019). 